\title{
Evaluation of risk factors' importance on adverse pregnancy and neonatal outcomes in women aged 40 years or older
}

\author{
Gunilla Sydsjö ${ }^{1 *}$ D, Malin Lindell Pettersson', Marie Bladh', Agneta Skoog Svanberg², Claudia Lampic ${ }^{3,4}$ and
} Elizabeth Nedstrand ${ }^{1}$

\begin{abstract}
Background: Women of advanced age (40 years or older) are generally, at risk for pregnancy and delivery related problems. In addition, there is limited knowledge on being of advanced age and having been given Assisted Reproductive Treatment (ART) and its association with negative obstetric outcomes. Therefore, data from the Swedish Medical Birth Register was used to investigate pregnancy and neonatal outcomes for women aged 40 or more who had given birth. The secondary aim was to compare the obstetric outcomes of women who had used ART and women who had not undergone ART while adjusting for marital status across the age groups.

Method: Women of advanced age who had given birth in Sweden during 2007-2012 formed the index group, $n=37,558$; a reference group of women comprised 71,472 women under the age of 40 . An additional subgroup of women aged 45 or older when giving birth was also formed, $n=2229$. The obstetric and neonatal data for all the women was derived from national register data.

Results: Women of advanced age were more often single, had undergone ART, and more often experienced adverse obstetric outcomes than did younger women. The neonate's health was also more often adversely affected expressed as being born with low birth weight and Small for Gestational Age (SGA), having lower Apgar scores, and having more health problems during the first week compared to the reference group.

Conclusions: Women who are approaching the upper limit of fecundity are at greater risk for having children who are preterm and SGA. The adverse effects of being preterm and SGA may have negative longterm effects, not only on the children but also on the mothers. This needs to be addressed more frequently in a clinical setting when advising women of all ages on pregnancy and ART treatment.
\end{abstract}

Keywords: Advanced maternal age, Pregnancy, Delivery, Neonate status

\section{Background}

During the past three decades there has been an increase in the percentage of women in the Western world who delay childbirth $[1,2]$. The percentage of women over 40 and even 45 years of age, who have given birth, has been increasing steadily. In Sweden, the percentage of women who are childless until they are 35 years or older has increased four-fold between 1984 and 2014 [3, 4]; during

\footnotetext{
* Correspondence: Gunilla.Sydsjo@liu.se

${ }^{1}$ Department of Obstetrics and Gynaecology, and Department of Clinical and Experimental Medicine, Linköping University, Linköping, Sweden. Linköping University, SE-581 85 Linköping, Sweden

Full list of author information is available at the end of the article
}

the same period the number of first-time mothers over 40 years of age increased from 170 to 1199 , i.e. a seven-fold increase [3]. The use of assisted reproductive technology (ART) in the form of gamete donation, embryo donation and In Vitro Fertilization (IVF), is an available choice in many countries and the possibilities for older women to become pregnant and to form a family have therefore become greater.

Women giving birth at an advanced age, defined as age 40 or older, are at an increased risk of complications during pregnancy and delivery, such as gestational diabetes and preeclampsia, compared to younger women [5]. They are also more prone to having children with a

(c) The Author(s). 2019 Open Access This article is distributed under the terms of the Creative Commons Attribution 4.0 International License (http://creativecommons.org/licenses/by/4.0/), which permits unrestricted use, distribution, and reproduction in any medium, provided you give appropriate credit to the original author(s) and the source, provide a link to the Creative Commons license, and indicate if changes were made. The Creative Commons Public Domain Dedication waiver (http://creativecommons.org/publicdomain/zero/1.0/) applies to the data made available in this article, unless otherwise stated. 
low or high birth weight [6], a caesarean delivery compared to younger women [7]. Moreover, their children are more often born moderately or very preterm, small for gestational age (SGA), with low Apgar scores, exhibiting fetal distress and even fetal and neonatal death [5, 7-12]. The risks associated with pregnancy and delivery in women of advanced age tend to remain higher than for younger women even after adjusting for socio-demographic factors [7].

In Sweden, couples and single women can use ART to conceive, several different options are available including IVF with their own gametes or oocyte-/sperm- donation, until the age of 42. Embryo donation was prohibited until the end of 2018 and treatment of single women has only recently been allowed [13]. Because of these restrictions, some Swedish women and couples have travelled abroad to have embryo-, oocyte- and/or sperm- donation. We have little knowledge about pregnancy and delivery outcomes for older Swedish women, those who are aged 40 or older and have received ART. In Sweden most IVF clinics do not provide ART for this group of women, so many women in this group who have used ART have done so outside of Sweden. However, several studies have shown that increasing maternal age as well as being single increases the risk for adverse pregnancy and delivery outcomes [14-16]. Also, using ART to achieve pregnancy has been shown to increase the risk for adverse pregnancy and delivery outcomes [15]. Thus, one can hypothesize that using ART to achieve pregnancy for older single woman may impose even greater risks for adverse outcomes.

Thus, the primary aim of this study was to investigate pregnancy and neonatal outcomes for women of advanced age. The secondary aim was to compare the obstetric outcomes of women who had used ART and women who had not used ART while adjusting for marital status across the age groups.

\section{Methods}

This study was designed as a case control study where data were obtained from the Medical Birth Register (MBR). As there is evidence in the literature that women at or over 40 years of age constitute an obstetric risk group, we chose this age cut-off for defining women of advanced age. Women 40 years of age or older who gave birth in Sweden between 2007 and 2013 formed the index group, $n=37,558$. This group was compared to a matched control group of women under the age of 40 who also gave birth during the same time period, $n=71,472$. The matching criteria were parity and year of birth. A subgroup of women aged 45 years or older when they gave birth was also formed, $n=2229$ (of $37,558,5.9 \%)$. The only exclusion criterion for this study was that none of the index women could serve as a control to themselves.

\section{The Swedish medical birth register}

The Swedish Medical Birth Register (MBR) is held by the Swedish National Board and Health and Welfare and contains medical information on practically all deliveries in Sweden from 1973 until the present [17].

\section{Measures}

This study used several different measures that can be divided into three main categories: socio-demographic and medical data (including civil status, employment, tobacco use, BMI, chronic illness, parity, and use of ART), pregnancy and delivery data (pre-eclampsia/eclampsia,

Table 1 Variable definitions

\begin{tabular}{|c|c|}
\hline Variable & Categories \\
\hline Maternal age & $\begin{array}{l}\leq 39 \text { years of age, } 40-44 \text { years of age, } \\
\geq 45 \text { years of age }\end{array}$ \\
\hline Civil status & Married/cohabiting, Single \\
\hline Employment & $\begin{array}{l}\text { Unemployed/student/other, Full time } \\
\text { employment, Part time employment }\end{array}$ \\
\hline Maternal BMI & $\begin{array}{l}\text { Normal, Underweight, Overweight, } \\
\text { Obese }\end{array}$ \\
\hline Tobacco use & No, Yes \\
\hline Chronic illness & No, Yes \\
\hline ART & No, Yes \\
\hline Pre-eclampsia/eclampsia & No, Yes \\
\hline Gestational diabetes & No, Yes \\
\hline Gestational hypertension & No, Yes \\
\hline Premature contractions & No, Yes \\
\hline Infection during pregnancy & No, Yes \\
\hline $\begin{array}{l}\text { Haemmorhage } \\
\text { (pre-, intra-, and post-partum) }\end{array}$ & No, Yes \\
\hline Paracervical block & No, Yes \\
\hline Pethidine & No, Yes \\
\hline Pudendal block & No, Yes \\
\hline Epidural & No, Yes \\
\hline Mode of delivery & $\begin{array}{l}\text { Normal delivery, Elective caesarean, } \\
\text { Emergency caesarean, Instrumental } \\
\text { delivery }\end{array}$ \\
\hline Gestational age & $\begin{array}{l}\text { Very preterm, }<32 \text { weeks, Preterm, } \\
32-36 \text { weeks, At term, 37-42 weeks, Post } \\
\text { term, }>42 \text { weeks }\end{array}$ \\
\hline Birthweight & $\begin{array}{l}\text { Normal birthweight, }>=2500 \mathrm{~g} \text {, Low } \\
\text { birthweight, } 1500-2499 \mathrm{~g} \text {, Very low } \\
\text { birthweight, }<1500 \mathrm{~g}\end{array}$ \\
\hline Size for gestational age & $\begin{array}{l}\text { Appropriate for gestational age (AGA), } \\
\text { Small for gestational age (SGA), Large for } \\
\text { gestational age (LGA) }\end{array}$ \\
\hline Apgar, 5 min & $0-6,7-10$ \\
\hline Apgar, 10 min & $0-6,7-10$ \\
\hline Child's health at delivery & Healthy - Yes, Healthy - No \\
\hline Neonatal survival & $0-27$ days, $>=28$ days \\
\hline
\end{tabular}


gestational diabetes, gestational hypertension, premature contractions, infections during pregnancy, haemorrhage pre-, intra- and post-partum, mode of delivery, and anaesthetic used during delivery) and neonatal data (birth weight, twinning, gestational age, size for gestational age, Apgar, child's overall health at birth and survival rate first month). The two latter categories constituted the outcome measures of interest. Definitions of all variables included in the study can be found in Table 1 .

\section{Statistical analysis}

The relationship between age when becoming a mother and socio-demographic data, pregnancy, delivery data, as well as neonatal data was initially analyzed using Pearson's Chi-square statistic and Student's t-test for continuous outcomes. Multivariate analyses included linear regression for continuous outcomes, such as gestational age, birthweight and the child's length, while multiple logistic regression was used for dichotomous outcomes (presence of pre-eclampsia/eclampsia, gestational diabetes, gestational hypertension, healthy child, survival during the first four weeks). In addition, data was stratified by age groups in order to analyze the relationship between type of pregnancy (ART vs. spontaneous) and civil status, gestational age, birthweight, and size for gestational age. All analyses were adjusted for civil status, mother's BMI, and indicator for ART/ not ART. In most of the variables some partial missing data was present. Therefore, in the analyses only data with complete observations have been included. A $p$-value $<0.05$ (two-sided) was considered statistically significant. All statistical analyses were performed using IBM SPSS, version 23 (IBM SPSS Inc., Armonk, NY).

\section{Ethical approval}

This study was approved by the Regional Ethical Review Board, Linköping, Sweden and was performed according

Table 2 Socio-demographic and medical background data on the study population

\begin{tabular}{|c|c|c|c|c|c|c|c|c|c|c|}
\hline & \multirow{2}{*}{\multicolumn{2}{|c|}{$\begin{array}{l}\text { Mothers } \leq 39 \\
n=71,472 \\
\text { min-max (mean/sd) }\end{array}$}} & \multirow{2}{*}{\multicolumn{2}{|c|}{$\begin{array}{l}\text { Mothers 40-44 } \\
n=35,329 \\
\text { min-max (mean/sd) }\end{array}$}} & \multirow{2}{*}{\multicolumn{2}{|c|}{$\begin{array}{l}\text { Mothers } \geq 45 \\
n=2229 \\
\text { min-max (mean/sd) }\end{array}$}} & \multirow[t]{2}{*}{ P-value ${ }^{1}$} & \multirow[t]{2}{*}{$P$-value ${ }^{2}$} & \multirow[t]{2}{*}{$P$-value ${ }^{3}$} & \multirow[t]{2}{*}{$P$-value ${ }^{4}$} \\
\hline & & & & & & & & & & \\
\hline \multirow[t]{2}{*}{ Maternal age, min-max (mean/sd) } & \multicolumn{2}{|c|}{$14-39(31.45 / 4.54)$} & \multicolumn{2}{|c|}{$40-44(41.41 / 1.19)$} & \multicolumn{2}{|c|}{$45-55(45.92 / 1.39)$} & $<0.001$ & $<0.001$ & $<0.001$ & $<0.001$ \\
\hline & $\mathrm{n}$ & $\%$ & $n$ & $\%$ & $n$ & $\%$ & $P$-value ${ }^{1}$ & $P$-value ${ }^{2}$ & P-value ${ }^{3}$ & P-value ${ }^{4}$ \\
\hline Civil status & & & & & & & $<0.001$ & $<0.001$ & $<0.001$ & $<0.001$ \\
\hline Married/ cohabiting & 64,059 & 89.6 & 30,106 & 85.0 & 1802 & 80.8 & & & & \\
\hline Single status at registration & 7413 & 10.4 & 5323 & 15.0 & 427 & 19.2 & & & & \\
\hline Parity & & & & & & & 0.001 & $<0.001$ & 0.664 & 0.126 \\
\hline Primiparous & 15,660 & 21.9 & 7417 & 20.9 & 497 & 22.3 & & & & \\
\hline Multiparous & 55,812 & 78.1 & 28,012 & 79.1 & 1732 & 77.7 & & & & \\
\hline Worked during pregnancy & & & & & & & $<0.001$ & $<0.001$ & $<0.001$ & 0.004 \\
\hline Unemployed/ student/ other & 17,103 & 27.4 & 6418 & 20.8 & 454 & 23.9 & & & & \\
\hline Full time & 27,543 & 44.2 & 16,219 & 52.5 & 964 & 50.8 & & & & \\
\hline Part time & 17,717 & 28.4 & 8267 & 26.8 & 478 & 25.2 & & & & \\
\hline Tobacco use during pregnancy & & & & & & & $<0.001$ & $<0.001$ & 0.004 & 0.383 \\
\hline Yes & 8329 & 12.3 & 3588 & 10.8 & 210 & 10.2 & & & & \\
\hline Mother's BMI (registration) & & & & & & & $<0.001$ & $<0.001$ & $<0.001$ & 0.003 \\
\hline Normal & 35,306 & 56.2 & 16,156 & 53.2 & 905 & 48.9 & & & & \\
\hline Underweight & 1165 & 1.9 & 291 & 1.0 & 21 & 1.1 & & & & \\
\hline Overweight & 17,117 & 27.3 & 9338 & 30.8 & 611 & 33.0 & & & & \\
\hline Obese & 9185 & 14.6 & 4566 & 15.0 & 314 & 17.0 & & & & \\
\hline Chronic illness $^{b}$ & & & & & & & 0.725 & 0.890 & 0.440 & 0.422 \\
\hline Yes & 15,035 & 21.0 & 7440 & 21.0 & 484 & 21.7 & & & & \\
\hline ART & & & & & & & $<0.001$ & $<0.001$ & $<0.001$ & $<0.001$ \\
\hline Yes & 1894 & 2.6 & 2813 & 7.9 & 249 & 11.2 & & & & \\
\hline
\end{tabular}

${ }^{a}$ Numbers may not add up to the total due to partially missing data

be.g. Diabetes, Hypertension, Arthritis, SLE, Kidney disease, Epilepsy, Asthma, Ulcerative colitis

${ }^{1}$ Across all age groups

$2 \leq 39$ vs. $40-44$

$3 \leq 39$ vs. $\geq 45$

${ }^{4} 40-44$ vs. $\geq 45$ 
to the Declaration of Helsinki. No 2014/111-31. Date: 26-03-2016.

\section{Results}

The older the women were the more likely they were to have been single at the time of the child's birth. Women between 40 and 44 years of age were $50 \%$ more likely to be single, while women aged 45 years or more were twice as likely to be single compared to women younger than 40. Women of advanced age were also more likely to be overweight or obese, and to have conceived using ART, Table 2. Moreover, the likelihood for having either pre-eclampsia/eclampsia or gestational diabetes increased with the mother's age when giving birth, Table 2. Also, with increasing age the proportion of women having an elective caesarean section increased from $7.5 \%$ among the youngest (below 40 years of age) to $20.4 \%$ among the oldest (45 years or older), Table 3.

Having a child born either preterm or very preterm was close to being twice as likely among women aged 45 or more compared to women below the age of 40 , Table 4. Women of advanced age were also twice as likely to deliver a twin or a child with low or very low birthweight. Moreover, the oldest mothers were also at a significantly higher risk for delivering a child with low Apgar, at 5 and $10 \mathrm{~min}$, a child who was not

Table 3 Pregnancy and delivery outcomes for the study population

\begin{tabular}{|c|c|c|c|c|c|c|c|c|c|c|}
\hline & \multicolumn{2}{|c|}{ Mothers $\leq 39 n=71,472$} & \multicolumn{2}{|c|}{ Mothers $40-44 n=35,429$} & \multicolumn{2}{|c|}{ Mothers $\geq 45 n=2229$} & \multirow[t]{2}{*}{$p$-value ${ }^{1}$} & \multirow[t]{2}{*}{$p$-value ${ }^{2}$} & \multirow[t]{2}{*}{$p$-value } & \multirow[t]{2}{*}{$p$-value } \\
\hline & $\mathrm{n}$ & $\%$ & $\mathrm{n}$ & $\%$ & $\bar{n}$ & $\%$ & & & & \\
\hline Pre-eclampsia/eclampsia & & & & & & & $<0.001$ & $<0.001$ & $<0.001$ & $<0.001$ \\
\hline Yes & 1776 & 2.5 & 1365 & 3.9 & 146 & 6.6 & & & & \\
\hline Gestational diabetes & & & & & & & $<0.001$ & $<0.001$ & $<0.001$ & $<0.001$ \\
\hline Yes & 935 & 1.3 & 917 & 2.6 & 86 & 3.9 & & & & \\
\hline Gestational hypertension & & & & & & & $<0.001$ & $<0.001$ & $<0.001$ & 0.017 \\
\hline Yes & 679 & 1.0 & 549 & 1.5 & 49 & 1.2 & & & & \\
\hline Premature contractions & & & & & & & 0.008 & 0.011 & 0.055 & 0.169 \\
\hline Yes & 229 & 0.31 & 82 & 0.2 & 2 & 0.1 & & & & \\
\hline Infection & & & & & & & 0.966 & 0.815 & 0.925 & 0.873 \\
\hline Yes & 200 & 0.3 & 102 & 0.3 & 6 & 0.3 & & & & \\
\hline Haemorrhage, prepartum & & & & & & & 0.910 & 0.525 & 0.677 & 0.509 \\
\hline Yes & 25 & 0.0 & 11 & 0.0 & 1 & 0.0 & & & & \\
\hline Mode of delivery & & & & & & & $<0.001$ & $<0.001$ & $<0.001$ & $<0.001$ \\
\hline Caesarean section (elective) & 5328 & 7.5 & 4931 & 13.9 & 455 & 20.4 & & & & \\
\hline Caesarean section (emergency) & 4931 & 6.9 & 4249 & 12.0 & 370 & 16.6 & & & & \\
\hline Instrumental delivery ${ }^{b}$ & 3592 & 5.0 & 2255 & 6.4 & 118 & 5.3 & & & & \\
\hline Epidural & & & & & & & $<0.001$ & $<0.001$ & $<0.001$ & 0.001 \\
\hline Yes & 14,454 & 20.2 & 6809 & 19.2 & 363 & 16.3 & & & & \\
\hline Haemorrhage, intrapartum & & & & & & & $<0.001$ & $<0.001$ & $<0.001$ & $<0.001$ \\
\hline Yes & 1242 & 1.7 & 1206 & 3.4 & 124 & 5.5 & & & & \\
\hline Haemorrhage, postpartum & & & & & & & $<0.001$ & $<0.001$ & $<0.001$ & 0.010 \\
\hline Yes & 3013 & 4.2 & 1658 & 4.7 & 131 & 5.9 & & & & \\
\hline Paracervical block ${ }^{a}$ & & & & & & & 0.571 & 0.296 & 0.803 & 0.973 \\
\hline Yes & 922 & 1.5 & 371 & 1.4 & 20 & 1.4 & & & & \\
\hline Pethidine $^{a}$ & & & & & & & 0.019 & 0.013 & 0.258 & 0.063 \\
\hline Yes & 1738 & 2.8 & 667 & 2.5 & 47 & 3.3 & & & & \\
\hline Pudendal block ${ }^{a}$ & & & & & & & $<0.001$ & $<0.001$ & 0.402 & 0.617 \\
\hline Yes & 1446 & 2.4 & 771 & 2.9 & 38 & 2.7 & & & & \\
\hline
\end{tabular}

\footnotetext{
${ }^{\mathrm{a}}$ Women delivered by caesarean section were excluded
}

${ }^{b}$ Vaginal delivery where either thongs or vacuum extraction was used

${ }^{1}$ Across all age groups, Person's chi-square

${ }^{2} \leq 39$ vs. $40-44$, Pearson's chi-square

${ }^{3} \leq 39$ vs. $\geq 45$, Pearson's chi-square

${ }^{4} 40-44$ vs. $\geq 45$, Pearson's chi-square 
Table 4 Neonatal birth characteristics for the three study groups ${ }^{a}$

\begin{tabular}{|c|c|c|c|c|c|c|c|c|c|c|}
\hline & \multirow{2}{*}{\multicolumn{2}{|c|}{$\begin{array}{l}\text { Mothers } \leq 39 \\
n=71,472 \\
\text { mean/SD }\end{array}$}} & \multirow{2}{*}{\multicolumn{2}{|c|}{$\begin{array}{l}\text { Mothers } 40-45 \\
n=35,429 \\
\text { mean/SD }\end{array}$}} & \multirow{2}{*}{\multicolumn{2}{|c|}{$\begin{array}{l}\text { Mothers } \geq 45 \\
n=2229 \\
\text { mean/SD }\end{array}$}} & \multirow{2}{*}{$\begin{array}{l}\text { CHI-SQUARE/ANOVA } \\
p \text {-value }\end{array}$} & \multirow[b]{2}{*}{$p$-value 2} & \multirow[b]{2}{*}{$p$-value ${ }^{3}$} & \multirow[b]{2}{*}{$p$-value } \\
\hline & & & & & & & & & & \\
\hline Child's length, cm (mean/SD) & \multirow{2}{*}{\multicolumn{2}{|c|}{$\begin{array}{l}50.34 / 2.65 \\
3541.49 / 604.21\end{array}$}} & \multicolumn{2}{|c|}{$50.27 / 2.85$} & \multicolumn{2}{|c|}{$49.85 / 3.30$} & $<0.001^{b}$ & $<0.001^{b}$ & $<0.001^{b}$ & $<0.001^{b}$ \\
\hline \multirow[t]{2}{*}{ Child's weight, gram (mean/SD) } & & & \multicolumn{2}{|c|}{$3507.06 / 639.68$} & \multicolumn{2}{|c|}{$3403.80 / 706.41$} & $<0.001^{b}$ & $<0.001^{\mathrm{b}}$ & $<0.001^{\mathrm{b}}$ & $<0.001^{\mathrm{b}}$ \\
\hline & $\mathrm{n}$ & $\%$ & $\mathrm{n}$ & $\%$ & $\mathrm{n}$ & $\%$ & $p$-value ${ }^{1}$ & $p$-value ${ }^{2}$ & $p$-value ${ }^{3}$ & $p$-value \\
\hline Twin & & & & & & & $<0.001$ & 0.007 & $<0.001$ & $<0.001$ \\
\hline No & 68,452 & 95.8 & 34,056 & 96.1 & 2033 & 91.2 & & & & \\
\hline Yes & 3020 & 4.2 & 1373 & 3.9 & 196 & 8.8 & & & & \\
\hline Gestational age & & & & & & & $<0.001$ & $<0.001$ & $<0.001$ & $<0.001$ \\
\hline Very preterm, $<32$ weeks & 680 & 1.0 & 436 & 1.2 & 54 & 2.4 & & & & \\
\hline Preterm, 32-36 weeks & 3870 & 5.4 & 2156 & 6.1 & 187 & 8.4 & & & & \\
\hline Post term, $>42$ weeks & 245 & 0.3 & 127 & 0.4 & 8 & 0.4 & & & & \\
\hline Birthweight & & & & & & & $<0.001$ & $<0.001$ & $<0.001$ & $<0.001$ \\
\hline Low birthweight, $1500 \mathrm{~g}-2499 \mathrm{~g}$ & 2633 & 3.7 & 1558 & 4.4 & 153 & 6.9 & & & & \\
\hline Very low birthweight, < $1500 \mathrm{~g}$ & 534 & 0.7 & 405 & 1.1 & 48 & 2.2 & & & & \\
\hline Size for gestational age & & & & & & & $<0.001$ & $<0.001$ & $<0.001$ & 0.884 \\
\hline$A G A^{5}$ & 67,107 & 93.9 & 32,710 & 92.3 & 2052 & 92.1 & & & & \\
\hline$S G A^{6}$ & 1281 & 1.8 & 987 & 2.8 & 63 & 2.8 & & & & \\
\hline $\mathrm{LGA}^{7}$ & 3084 & 4.3 & 1732 & 4.9 & 114 & 5.1 & & & & \\
\hline Apgar 5 & & & & & & & $<0.001$ & $<0.001$ & $<0.001$ & 0.168 \\
\hline Low score, 0-6 & 763 & 1.1 & 546 & 1.6 & 43 & 1.9 & & & & \\
\hline Apgar 10 & & & & & & & $<0.001$ & $<0.001$ & $<0.001$ & 0.015 \\
\hline Low score0-6 & 253 & 0.4 & 181 & 0.5 & 20 & 0.9 & & & & \\
\hline Child health ${ }^{c}$ & & & & & & & $<0.001$ & $<0.001$ & $<0.001$ & $<0.001$ \\
\hline Healthy - No & 14,282 & 20.4 & 8422 & 24.3 & 602 & 27.7 & & & & \\
\hline Survival & & & & & & & $<0.001$ & $<0.001$ & 0.011 & 0.328 \\
\hline $0-27$ days & 123 & 0.2 & 102 & 0.3 & 9 & 0.4 & & & & \\
\hline
\end{tabular}

a Student's t-test

${ }^{b}$ Numbers may not add up to the total due to partially missing data

'Defined as: Healthy child, examined at the delivery ward

${ }^{1}$ Across all age groups

${ }^{2} \leq 39$ vs. $40-44$, Pearson's chi-square

${ }^{3} \leq 39$ vs. $\geq 45$, Pearson's chi-square

${ }^{4} 40-44$ vs. $\geq 45$, Pearson's chi-square

${ }^{5}$ Appropriate for gestational age

${ }^{6}$ Small for gestational age

${ }^{7}$ Large for gestational age

well as a neonate, and to have children more likely to die within the first four weeks after delivery, Table 4.

Data were further stratified by method of conception, in order to investigate the differences in birth characteristics and civil status in relation to age when becoming a mother in each of these groups. The analysis showed that in both groups (ART and spontaneous pregnancy) women of advanced age were more prone to be single, delivering a child preterm or with low birthweight as well as an SGA child, compared to women younger than 40 (Table 5). Furthermore, the multiple logistic regression, where data was stratified by age, it was found that women who had used ART to achieve pregnancy were more likely to be single, and to give birth to a preterm child in all age groups. Moreover, women of advanced age had an increased likelihood of delivering a very preterm child (Table 6).

The multivariate analyses (covariates included were civil status, method of conception, mother's BMI in early pregnancy, and mother's age when giving birth) revealed that being single or underweight, having an ART treatment, and being older were all factors negatively related to birthweight, child's length at birth as well as gestational age (Table 7). In the multiple 
Table 5 Birth characteristics and civil status by maternal age when giving birth and type of pregnancy ${ }^{\text {a }}$

\begin{tabular}{|c|c|c|c|c|c|c|c|c|}
\hline & ART & & & & Spontaneous & & & \\
\hline & $\leq 39$ & $40-44$ & $\geq 45$ & $p$-value ${ }^{b}$ & $\leq 39$ & $40-44$ & $\geq 45$ & $p$-value \\
\hline & $n(\%)$ & $n(\%)$ & $n(\%)$ & & $n(\%)$ & $n(\%)$ & $n(\%)$ & \\
\hline Civil status & & & & $<0.001$ & & & & $<0.001$ \\
\hline Married/ cohabiting & $1859(98.2)$ & $2651(94.2)$ & $227(91.2)$ & & $62,200(89.4)$ & $27,455(84.2)$ & $1575(79.5)$ & \\
\hline Single status at registration & $35(1.8)$ & $162(5.8)$ & $22(8.8)$ & & $7378(10.6)$ & $5161(15.8)$ & $405(20.5)$ & \\
\hline Parity & & & & & & & & $<0.001$ \\
\hline Primiparous & $872(46.0)$ & $1318(46.9)$ & $120(48.2)$ & 0.754 & $14,788(21.3)$ & $6099(18.7)$ & $377(19.0)$ & \\
\hline Multiparous & $1022(54.0)$ & $1495(53.1)$ & $129(51.8)$ & & $54,790(78.7)$ & $26,517(81.3)$ & $1603(81.0)$ & \\
\hline Twin & & & & $<0.001$ & & & & $<0.001$ \\
\hline No & $1404(74.1)$ & $2381(84.6)$ & $170(68.3)$ & & $67,048(96.4)$ & $31,675(97.1)$ & $1863(94.1)$ & \\
\hline Yes & $490(25.9)$ & $432(15.4)$ & $79(31.7)$ & & $2530(3.6)$ & $941(2.9)$ & $117(5.9)$ & \\
\hline Gestational age & & & & $<0.001$ & & & & $<0.001$ \\
\hline At term, 37-42 weeks & $1556(82.2)$ & $2454(87.2)$ & $190(76.3)$ & & $65,066(93.6)$ & $30,223(92.8)$ & $1787(90.4)$ & \\
\hline Post term, $>42$ weeks & $4(0.2)$ & $11(0.4)$ & $1(0.4)$ & & $241(0.3)$ & $116(0.4)$ & $7(0.4)$ & \\
\hline Preterm, 32-36 weeks & $277(14.6)$ & $284(10.1)$ & $49(19.7)$ & & $3593(5.2)$ & $1872(5.7)$ & $138(7.0)$ & \\
\hline Very preterm, $<32$ weeks & $57(3.0)$ & $64(2.3)$ & $9(3.6)$ & & $623(0.9)$ & $372(1.1)$ & $45(2.3)$ & \\
\hline Birthweight & & & & $<0.001$ & & & & $<0.001$ \\
\hline Normal birthweight, $\geq 2500 \mathrm{~g}$ & $1614(85.5)$ & $2526(90.0)$ & $193(77.8)$ & & $66,533(95.8)$ & $30,834(94.8)$ & $1831(92.6)$ & \\
\hline Low birthweight, $1500 \mathrm{~g}-2499 \mathrm{~g}$ & $230(12.2)$ & $228(8.1)$ & $45(18.1)$ & & $2403(3.5)$ & $1330(4.1)$ & $108(5.5)$ & \\
\hline Very low birthweight, < $1500 \mathrm{~g}$ & $44(2.3)$ & $52(1.9)$ & $10(4.0)$ & & $490(0.7)$ & $1330(1.1)$ & $38(1.9)$ & \\
\hline Size for gestational age & & & & 0.255 & & & & $<0.001$ \\
\hline$A G A^{1}$ & $1788(94.4)$ & $2637(93.7)$ & $226(90.8)$ & & $65,319(93.9)$ & $30,073(92.2)$ & $1826(92.2)$ & \\
\hline$S G A^{2}$ & $42(2.2)$ & $72(2.6)$ & $10(4.0)$ & & $1239(1.8)$ & $915(2.8)$ & $53(2.7)$ & \\
\hline $\mathrm{LGA}^{3}$ & $64(3.4)$ & $104(3.7)$ & $13(5.2)$ & & $3020(4.3)$ & $1628(5.0)$ & $101(5.1)$ & \\
\hline
\end{tabular}

${ }^{a}$ Numbers may not add up to the total due to partially missing data

bPearson's chi-square

${ }^{1}$ Appropriate for gestational age

${ }^{2}$ Small for gestational age

${ }^{3}$ Large for gestational age

logistic regression models of pregnancy and delivery complications women of a higher age and BMI were found to have an increased odds ratio for hypertensive disease during pregnancy, gestational diabetes, pre-eclampsia/eclampsia, and having a child with registered health problems during the neonatal period (Table 7). Alternative multivariate models where twinning of the child was excluded were also considered. However, since twins are often born preterm and/or with a lower birth weight and that older women are more likely to have undergone an ART-treatment to achieve a pregnancy, which in itself is considered a risk factor for twinning, low birthweight, and prematurity, it was decided to keep the variable twinning in the final models. In a sub-group analysis of multiparous women, the findings of increased the risks for negative pregnancy and delivery outcomes among older women where verified (data not shown).

\section{Discussion}

The results reveal that women of advanced age were more often single, had used ART, and had adverse obstetric outcomes more often than did younger women. The neonates born to women of advanced age were more likely to have health problems including being underweight or SGA, having lower a Apgar, and having other additional health problems during the first week.

The strength of the study is the availability of register data on all the women who gave birth in Sweden during the study period and medical data that were reported in a standardized form, thus minimizing recall bias. One limitation in this study is that we had no information about the ART method used. It might be that for single women of relatively advanced age the use of embryo donation is the method used, whereas for cohabiting women of advanced age it might be more likely that they had used their partner's sperm and an oocyte donation. 
Table 6 Odds ratios for women in the different age groups who had given birth following ART vs. spontaneous pregnancy ${ }^{\mathrm{a}}$

\begin{tabular}{|c|c|c|c|c|c|}
\hline & \multicolumn{4}{|l|}{ Age group } & \multirow[t]{2}{*}{ All age groups } \\
\hline & $\leq 39$ & $40-44$ & $\geq 45$ & $\geq 40$ & \\
\hline & OR $(95 \% \mathrm{Cl})$ & OR $(95 \% \mathrm{Cl})$ & OR (95\% Cl) & OR $(95 \% \mathrm{Cl})$ & OR $(95 \% \mathrm{Cl})$ \\
\hline \multicolumn{6}{|l|}{ Civil status } \\
\hline Married/ cohabiting & Reference & Reference & Reference & Reference & Reference \\
\hline Single status at registration & $7.51(5.35-10.53)$ & $4.09(3.46-4.83)$ & $3.71(2.28-6.04)$ & $0.73(3.24-4.29)$ & $4.62(4.02-5.33)$ \\
\hline \multicolumn{6}{|l|}{ Parity } \\
\hline Primiparous & Reference & Reference & Reference & Reference & Reference \\
\hline Multiparous & $0.27(0.25-0.30)$ & $0.21(0.20-0.23)$ & $0.22(0.16-0.30)$ & $0.25(0.24-0.27)$ & $0.24(0.22-0.25)$ \\
\hline \multicolumn{6}{|l|}{ Twin } \\
\hline No & Reference & Reference & Reference & Reference & Reference \\
\hline Yes & $9.85(8.58-11.30)$ & $7.50(6.87-9.20)$ & $8.02(5.21-12.35)$ & $7.94(7.22-8.72)$ & $8.91(8.06-9.82)$ \\
\hline \multicolumn{6}{|l|}{ Age group } \\
\hline$\leq 39$ & NA & NA & NA & NA & Reference \\
\hline $40-44$ & NA & NA & NA & NA & $3.40(3.57-4.05)$ \\
\hline$\geq 45$ & NA & NA & NA & NA & $4.72(4.05-5.50)$ \\
\hline \multicolumn{6}{|l|}{ Gestational age } \\
\hline At term, 37-42 weeks & Reference & Reference & Reference & Reference & Reference \\
\hline Post term, $>42$ weeks & $0.63(0.23-1.72)$ & $0.92(0.49-1.75)$ & $1.65(0.19-14.31)$ & $0.90(0.54-1.50)$ & $0.86(0.51-1.44)$ \\
\hline Preterm, 32-36 weeks & $1.37(1.14-1.65)$ & $1.20(1.00-1.43)$ & $0.95(0.55-1.66)$ & $1.26(1.12-1.43)$ & $1.26(1.12-1.43)$ \\
\hline Very preterm, $<32$ weeks & $1.57(0.93-2.64)$ & $1.92(1.15-3.19)$ & $0.13(0.02-0.93)$ & $1.47(1.03-2.10)$ & $1.60(1.11-2.29)$ \\
\hline \multicolumn{6}{|l|}{ Birthweight } \\
\hline Normal birthweight, $\geq 2500 \mathrm{~g}$ & Reference & Reference & Reference & Reference & Reference \\
\hline Low birthweight, $1500 \mathrm{~g}-2499 \mathrm{~g}$ & $1.01(0.81-1.25)$ & $0.90(0.72-1.11)$ & $1.62(0.88-2.99)$ & $1.02(0.88-1.17)$ & $0.98(0.85-1.14)$ \\
\hline Very low birthweight, < $1500 \mathrm{~g}$ & $0.81(0.56-1.45)$ & $0.59(0.34-1.02)$ & $4.82(0.67-34.83)$ & $0.82(0.56-1.20)$ & $0.70(0.47-1.04)$ \\
\hline \multicolumn{6}{|l|}{ Size for gestational age } \\
\hline$A G A^{1}$ & Reference & Reference & Reference & Reference & Reference \\
\hline$S G A^{2}$ & $1.35(0.97-1.88)$ & $0.84(0.64-1.10)$ & $1.54(0.69-3.42)$ & $1.16(0.96-1.42)$ & $1.02(0.83-1.24)$ \\
\hline $\operatorname{LGA}^{3}$ & $1.27(0.98-1.64)$ & $1.08(0.88-1.32)$ & $1.70(0.92-3.16)$ & $1.22(1.05-1.43)$ & $1.17(1.00-1.37)$ \\
\hline
\end{tabular}

${ }^{a}$ Adjusting for all variables presented in Table 4

${ }^{1}$ Appropriate for gestational age

${ }^{2}$ Small for gestational age

${ }^{3}$ Large for gestational age

In the MBR register there are also limited data on the women's health status. ART treatment may have been underreported in all study groups; we have not been able to control for this through examination of registers on IVF pregnancies done in Sweden. Also, among women who have received treatment abroad underreporting is likely since there was no obligation to report ART to the midwife and obstetricians in the antenatal setting.

Our results correspond with results from other studies on older women's health status and obstetric outcomes $[7,10,12]$. The reason for these outcomes might be multifactorial. One important factor might be the use of ART and the other factor the woman's biological age. The woman's fecundity clearly decreases with increasing age, generally more noticeably a few years after age 30 , to cease about 10 years before menopause [18].

There is only limited information on the use of ART for women in advanced age groups and the information is often available only through case reports in which there is no information on the specific technique used [19].

The risks for the mother and the neonate with gamete donation and embryo donation are not fully understood and have not yet been thoroughly investigated. There seems to be a consensus that the risk for women to have pregnancy complications such as preeclampsia and bleeding and the risk for the neonate to be born prematurely or with low birth weight is of considerable importance $[20,21]$. Having premature and small for 


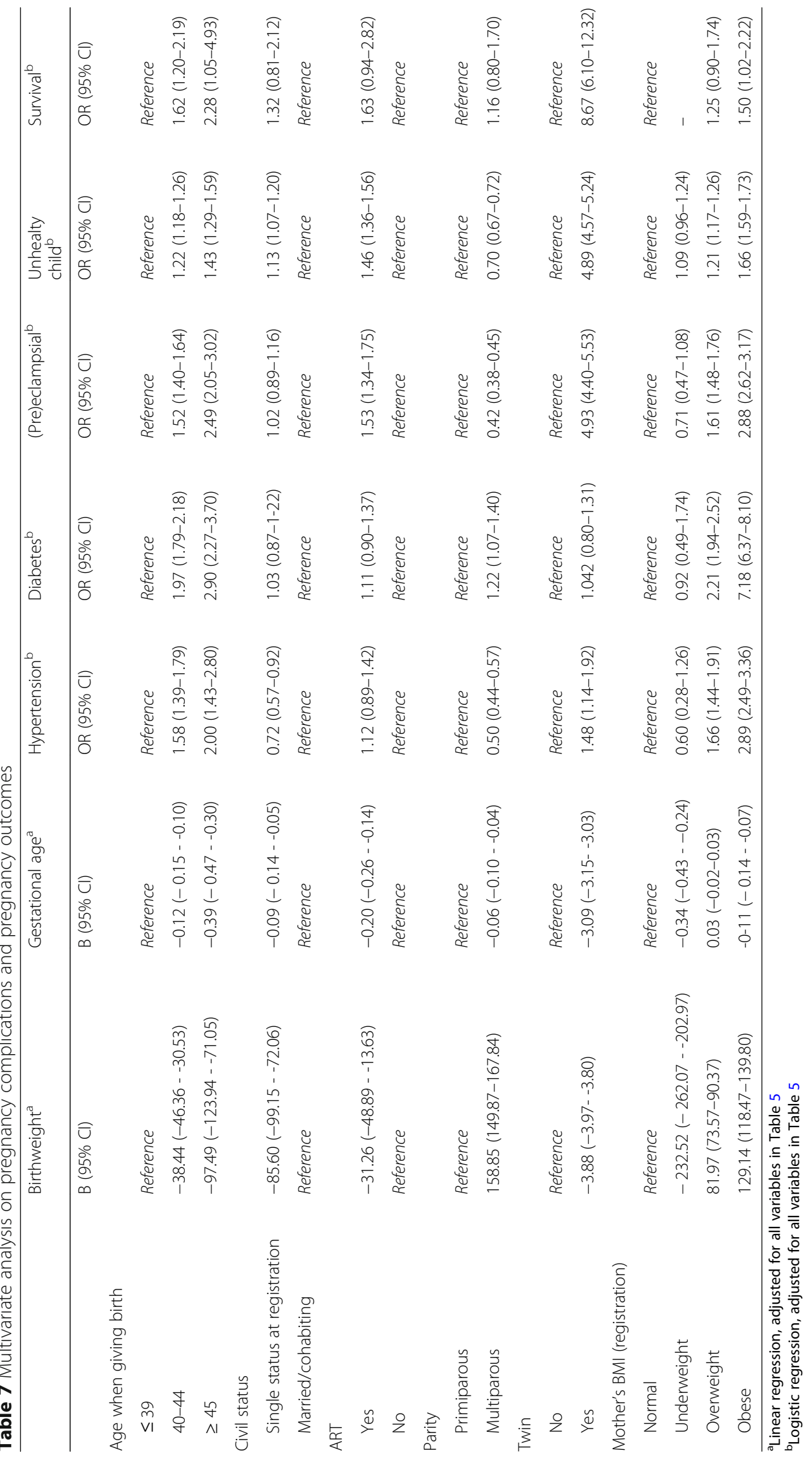


gestational children might also affect the mother and family mentally, as well as economically and socially, both in long-term as well as in short-term [22, 23].

For women who have given birth at a relatively advanced age there is still no consensus in the medical literature on the specific age or age range when the risk elevates significantly; some claim that only after 40 years of age there are risk of clinical significance but others report that the risk is already clinically significant at 35 years [24, 25]. In a Norwegian cohort of over 40,000 women who all answered a questionnaire at around gestational week 17, the authors found that women older than 38 years experienced problems related to physical aging including hypertension, back and shoulder problems, and diabetes. Furthermore, these women were more likely to have mental health problems than younger women [26].

Women in general might not be aware of obstetric and delivery risks for older women, and women who seek treatment abroad with ART and with donated gametes might be especially optimistic. It also seems possible that society at large and some medical practitioners tend to be overly affirmative in an effort not to discriminate against older women. This may even be true of personnel at clinics offering treatment. For women treated in other settings, for example abroad in private clinics, there may be age limits different from those in Sweden or even no age limits at all. There are other risks related to ART treatment in women of advanced age who have been treated abroad such as undetected illnesses both mental and physical, since not all clinics have access to medical records and thus have a limited medical history or have access to complete medical history and ongoing illnesses. Some women may themselves be unaware of the consequences or may want to have a child no matter what risks.

The women who were single when giving birth seem to represent a group of women that have more medical problems during pregnancy and delivery than women who are not single. The findings in this study indicate that women who are of very advanced age (above 45 years of age), single and had used ART are most likely to be women who have gone abroad to have their treatment since they cannot be treated in Sweden.

The increased risks for older women giving birth have important clinical implications and professionals need to be fully aware of the risks they expose the older women to by letting them go through ART. For children who were born preterm and SGA there may be future long-term health problems that will require medical attention and care. The development and needs of children born in families where the parents are of advanced age have not yet been well studied.

\section{Conclusions}

In conclusion, the results from this study show that there is a substantial need to inform older, as well as younger, women about reproductive issues. This includes guidelines, both in general and in clinical practices, on becoming pregnant and whether to use or not to use ART. Women who are in the later stages of their reproductive life are at great risk for having children who are preterm and SGA. This may have long-term consequences not only for the children but also for the mothers. IVF clinics need to show a great medical awareness when offering older women treatment with their own gametes and with donated sperm and oocytes.

Society must also be more aware of the risks and to be more willing to discuss reproductive problems associated with advanced maternal age. For women and children, the need for future care is also a medical and societal matter that needs to be given more attention so that approaches are developed to serve these families both in the long as well as the short term.

\section{Acknowledgements \\ Professor Lawrence Lundgren for language editing.}

\section{Funding}

This study was supported in all stages by grants from The Research Council in the southeast of Sweden, FORSS.

\section{Availability of data and materials}

The Ethical Review Board approval was obtained for public sharing and presentation of data on group level only. This means that the data used in this study can only be used for the approved research and cannot be shared by the authors.

\section{Author's contributions}

GS, had the original idea for the study. MPL GS, MB, CL, ASS and EN planned the study. MB, MPL and GS analyzed the data. All authors contributed to the interpretation of the data and to the revisions, and gave input at all stages of the study. All authors have approved the final version of the article for publication.

\section{Competing interest}

The authors declare that they have no competing interests.

\section{Ethics approval and consent to participate}

The study was approved by the regional Ethical Review Board, Linköping, Sweden, no. 2014/111-31. Date: 26-03-2016. Informed consent is not applicable.

Informed consent was not required, according to national guidelines, since this is a register study with an ethical approval from the ethical review board and permission to use data was obtained from the register holders.

\section{Consent for publication}

Not applicable.

\section{Publisher's Note}

Springer Nature remains neutral with regard to jurisdictional claims in published maps and institutional affiliations.

\section{Author details}

'Department of Obstetrics and Gynaecology, and Department of Clinical and Experimental Medicine, Linköping University, Linköping, Sweden. Linköping University, SE-581 85 Linköping, Sweden. ²Department of Women's and Children's Health, Uppsala University, Uppsala, Sweden. ${ }^{3}$ Department of 
Neurobiology, Care Sciences and Society, Karolinska Institutet, S-141 83 Huddinge, Sweden. ${ }^{4}$ Department of Women's and Children's Health, Karolinska Institutet, S-171 77 Stockholm, Sweden.

Received: 2 May 2018 Accepted: 6 March 2019

Published online: 13 March 2019

\section{References}

1. Heffner LJ. Advanced maternal age--how old is too old? N Engl J Med. 2004;351(19):1927-9.

2. Matthews TJ, Hamilton BE. Delayed childbearing: more women are having their first child later in life. NCHS data brief. 2009;21:1-8.

3. National Board of Health and Welfare. Pregnancies, Deliveries and Newborn Infants The Swedish Medical Birth Register 1973-2014 Assisted Reproduction, treatment 1991-2013 [https://www.socialstyrelsen.se/Lists/ Artikelkatalog/Attachments/20009/2015-12-27.pdf].

4. Statistics, Sweden. In.; 2017

5. Jacobsson B, Ladfors L, Milsom I. Advanced maternal age and adverse perinatal outcome. Obstet Gynecol. 2004;104(4):727-33.

6. Jolly M, Sebire N, Harris J, Robinson S, Regan L. The risks associated with pregnancy in women aged 35 years or older. Hum Reprod. 2000;15(11): 2433-7.

7. Kenny LC, Lavender T, McNamee R, O'Neill SM, Mills T, Khashan AS. Advanced maternal age and adverse pregnancy outcome: evidence from a large contemporary cohort. PLoS One. 2013;8(2):e56583.

8. Waldenstrom U, Aasheim V Nilsen AB, Rasmussen S, Pettersson HJ, Schytt E. Adverse pregnancy outcomes related to advanced maternal age compared with smoking and being overweight. Obstet Gynecol. 2014;123(1):104-12.

9. Cavazos-Rehg PA, Krauss MJ, Spitznagel EL, Bommarito K, Madden T, Olsen MA, Subramaniam H, Peipert JF, Bierut LJ. Maternal age and risk of labor and delivery complications. Matern Child Health J. 2015;19(6):1202-11.

10. Waldenström U, Cnattingius S, Vixner L, Norman M. Advanced maternal age increases the risk of very preterm birth, irrespective of parity: a populationbased register study. BJOG. 2017;124(8):1235-44

11. Huang L, Sauve R, Birkett N, Fergusson D, van Walraven C. Maternal age and risk of stillbirth: a systematic review. CMAJ. 2008;178(2):165-72.

12. Blomberg $M$, Birch Tyrberg R, Kjolhede P. Impact of maternal age on obstetric and neonatal outcome with emphasis on primiparous adolescents and older women: a Swedish medical birth register study. BMJ Open. 2014; 4(11):e005840

13. Government S: Assisterad befruktning för ensamstående kvinnor. In: 2015/ 16:SoU3. Edited by Government S. Sweden; 2016

14. Ludford I, Scheil W, Tucker G, Grivell R. Pregnancy outcomes for nulliparous women of advanced maternal age in South Australia, 1998-2008. Aust N Z J Obstet Gynaecol. 2012:52(3):235-41

15. Schoen C, Rosen T. Maternal and perinatal risks for women over 44--a review. Maturitas. 2009;64(2):109-13.

16. Orellana PL. Increase in Preterm Birth during Demographic Transition in Chile from 1991 to 2012. Biomed Res Int. 2015;2015:4.

17. The Swedish Medical Birth Register: a summary of content and quality. [http://www.socialstyrelsen.se/Lists/Artikelkatalog/Attachments/10655/2003112-3_20031123.pdf].

18. te Velde $E R$, Pearson PL. The variability of female reproductive ageing. Hum Reprod Update. 2002;8(2):141-54.

19. De Brucker M, Tournaye H, Haentjens P, Verheyen G, Collins J, Camus M. Assisted reproduction counseling in women aged 40 and above: a cohort study. J Assist Reprod Genet. 2013;30(11):1431-8

20. Elenis E, Svanberg AS, Lampic C, Skalkidou A, Akerud H, Sydsjo G. Adverse obstetric outcomes in pregnancies resulting from oocyte donation: a retrospective cohort case study in Sweden. BMC Pregnancy Childbirth. 2015; 15:247.

21. Elenis E, Sydsjo G, Skalkidou A, Lampic C, Svanberg AS. Neonatal outcomes in pregnancies resulting from oocyte donation: a cohort study in Sweden. BMC Pediatr. 2016;16(1):170.

22. Lakshmanan AAM, Fleegler E, Kipke M, Friedlich PS, Mccormick MC, Belfort MB. The impact of pretem birth $<37$ weeks on parents and families: a crosssectional study in the 2 years after discharge from the neanatal intensive care unit. Health Qual Life Outcome. 2017:15(1):1-13.

23. Holditch-Davis D, Santos H, Levy J, White-Traut R, O'Shea TM, Geraldo V, David R. Patterns of psychological distress in mothers of preterm infants. Infant Behav Dev. 2015;41:154-63.
24. Delbaere I, Verstraelen H, Goetgeluk S, Martens G, De Backer G, Temmerman M. Pregnancy outcome in primiparae of advanced maternal age. Eur J Obstet Gynecol Reprod Biol. 2007;135(1):41-6.

25. Klemetti R, Gissler M, Sainio S, Hemminki E. At what age does the risk for adverse maternal and infant outcomes increase? Nationwide register-based study on first births in Finland in 2005-2014. Acta Obstet Gynecol Scand. 2016:95(12):1368-75

26. Nilsen $A B$, Waldenstrom $U$, Hjelmstedt A, Rasmussen $S$, Schytt $E$. Characteristics of women who are pregnant with their first baby at an advanced age. Acta Obstet Gynecol Scand. 2012;91(3):353-362x.
Ready to submit your research? Choose BMC and benefit from:

- fast, convenient online submission

- thorough peer review by experienced researchers in your field

- rapid publication on acceptance

- support for research data, including large and complex data types

- gold Open Access which fosters wider collaboration and increased citations

- maximum visibility for your research: over $100 \mathrm{M}$ website views per year

At $\mathrm{BMC}$, research is always in progress.

Learn more biomedcentral.com/submissions 